\title{
SDRE APPLIED TO POSITION AND VIBRATION CONTROL OF A ROBOT MANIPULATOR WITH A FLEXIBLE LINK
}

\author{
Jeferson José de lima, Angelo Marcelo Tusset, Frederic Conrad Janzen, \\ Vincius Piccirillo, Claudinor Bitencourt Nascimento \\ UTFPR,Ponta Grossa,PR,Brazil; email: jefersonjl82@gmail.com; tusset@utfpr.edu.br; fcjanzen@utfpr.edu.br; \\ piccirillo@utfpr.edu.br; claudinor@utfpr.edu.br \\ José MANOEL BALthazAR \\ UNESP, Bauru,SP, Brazil; email: jmbaltha@gmail.com
}

Reyolando Manoel Lopes Rebello da Fonseca Brasil

UFABC, Santo Andre, SP, Brazil; email: reyolando.brasil@ufabc.edu.br

\begin{abstract}
This paper presents position and vibration control of a flexible robot composed of two rigid and one flexible links. Position is controlled by the current applied to the DC motor armature. To control vibrations of the flexible structure, Shape Memory Alloys (SMA) are used. Due to phase transformations, the SMA can change its stiffness through temperature variation, considering and taking advantage of this characteristic the vibration control is done. Control is achieved via the State Dependent Ricatti Equations (SDRE) technique, which uses suboptimal control and system local stability search. The simulation results show the feasibility of the proposed control for the considered system.
\end{abstract}

Keywords: SDRE control, flexible robotic arm, SMA, active vibration control

\section{Introduction}

In the last decades, several researches on robotic manipulators with flexible links have come to attention due to their many advantages over rigid manipulators (Kalyoncu, 2008).

Shawky et al. (2013) remarked that flexible manipulator systems offer several advantages in contrast to the traditional rigid manipulator. The advantages include faster response, lower energy consumption, relatively smaller actuators and less overall mass and, in general, smaller final cost. Due to its flexible nature, control of these flexible systems takes into account both rigid body and elastic degrees of freedom. It is important to recognize the flexible nature of the manipulator and construct a mathematical model for the system that accounts for the interactions between the actuators and payload.

According to Banks et al. (1996) and Gabbert and Schulz (1996), it is necessary to improve robotic manipulators including the use of smart materials to assist control by changing the structural behavior.

There are several studies suggesting solutions to the problem of handlers with flexible elements such as (Sawada et al., 2004), which have been exploited for the purposes of efficiency and flexibility of a handler in microgravity environment. In Inifene (2007), the dynamics of a flexible link and a comparative study of some implemented control is shown. (Heidari et al., 2013) proposes a nonlinear finite element modeling of flexibility of handlers. In Grandinetti et al. (2012), a three-degree-of-freedom cylindrical manipulator system with a flexible link on its tip is studied and experimentally implemented, and the active vibration control is analyzed. In Halim et al. (2014), the use of a decentralized vibration control scheme for suppressing vibration of a multi-link flexible robotic manipulator using embedded smart piezoelectric transducers is 
investigated. In Pereira et al. (2012), an adaptive control applied to single link-flexible manipulators, utilizing a feedback controller of the joint angle to guarantee trajectory tracking of the joint angle with an adaptive input shaper updated by algebraic non-asymptotic identification is proposed.

Bottega et al. (2009) proposed a tracking control model for a flexible link robotic manipulator using simultaneously motor torques and piezoelectric actuators. Molter et al. (2010) used SDRE control technique design for flexible manipulators using piezoelectric actuators. In Shawky et al. (2013), the tip position of a one-link flexible manipulator was modeled and controlled. The control strategy was based on the SDRE control design method in the context of application to robotics. The experimental results revealed that the nonlinear SDRE controller was near optimal and robust, and its performance compared favorably to the conventional PD controller strategy.

This work proposes the control of a robotic manipulator for aerospace applications. It is composed of two rigid links modeled as proposed by Korayem et al. (2011), and one flexible link modeled as proposed by Janzen et al. (2014). The gravity effects are not considered, similar to the proposal presented by Sawada et al. (2004). In order to reduce vibrations of the flexible manipulator, it is proposed to use SMAs. Shape Memory Alloys (SMAs) are a group of metallic materials with the ability to return to a previously defined shape or size, after deformation, by applying a specific temperature. This effect occurs due to the shift in the materials crystalline structure between two different phases, called martensite and austenite (Piccirillo et al., 2008, 2009). When SMAs are heated, they present changes in crystal structure. These changes generate large forces that can be used in actuator systems (Ge et al., 2013). In this way, SMAs can be used in many application areas, like robotics, since they allow for a lighter weight manipulator and longer links, which are important for aerospace applications.

The proposed feedback control system is designed to take the system into a desired coordinate. The feedback control is obtained using SDRE. The SDRE control choice is because the control computer algorithm is simple and highly effective for nonlinear feedback control. It has been successfully used in robotic manipulators (Innocenti et al., 2000; Xin et al., 2001; Korayem et al., 2010; Nekoo, 2013; Korayem and Nekoo, 2014, 2015a), and in robotic manipulators with a flexible joint or/and with flexible link (Korayem et al. 2011; Fenili and Balthazar, 2011; Shawky et al., 2013; Lima et al., 2014; Korayem and Nekoo, 2015b).

The paper is organized is as follows. Section 2 provides the dynamic model for a two rigid and a flexible link manipulator. Section 3 presents the SDRE control project considering both control of just positioning, without vibration control of the flexible link, as in the case of vibration control of the flexible link by temperature variation (SMAs). Concluding remarks are given in Section 4.

\section{Mathematical model}

The adopted manipulator model consists of two rigid links and the third flexible one. The DC motors and joints of the robotic manipulator are presented in Fig. 1, and the transmission motor torque to the manipulator is given by Eq. (2.6),

The governing equations of motions are obtained through Lagrangian formalism. To derive the equations of motion of the manipulator, one needs the kinetic energy $\Im$ and the potential energy $\Gamma$ in the Lagrangian function $L$

$$
L=\Im-\Gamma
$$

Next, one must apply the equations of Euler-Lagrange (Lima et al., 2014)

$$
\frac{d}{d t}\left(\frac{\partial L}{\partial \dot{\theta}_{i}}\right)-\frac{\partial L}{\partial \theta_{i}}=\tau_{i}
$$




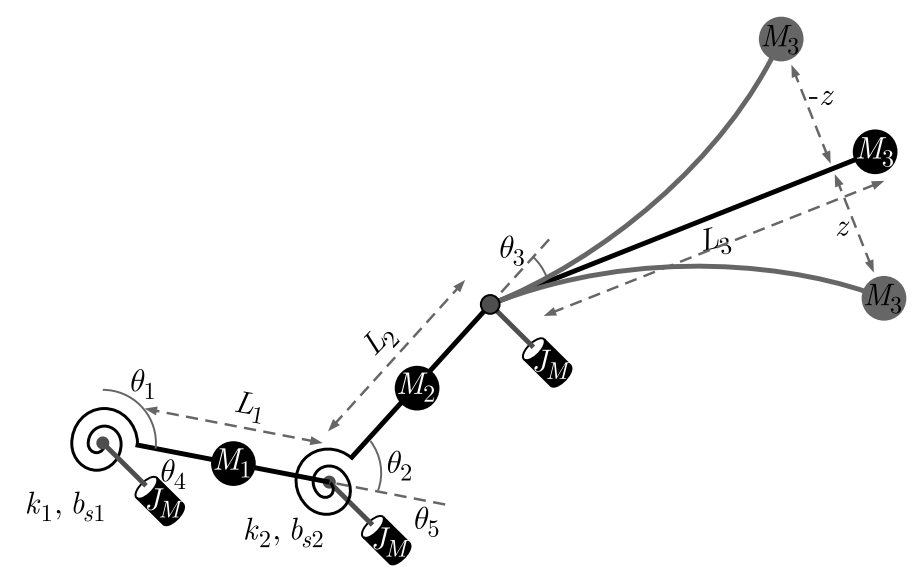

Fig. 1. Schematic of the manipulator model, where $\theta_{1,2,3}$ are the joint angles of each link, $\theta_{4,5}$ are the corresponding motor angles for the link, $b_{s}$ is the damping constant, $k$ is the spring constant, and the variable $z$ is the displacement of the beam end

The right hand side of Eq. (2.2) are non-conservative forces. Thus, we obtain Eq. (2.3), the equations of motion of the link (Korayem et al., 2011; Lima et al., 2014)

$$
\mathbf{M}(\boldsymbol{\theta}) \ddot{\boldsymbol{\theta}}=-\mathbf{C}(\boldsymbol{\theta}, \dot{\boldsymbol{\theta}})-\mathbf{F}(\dot{\boldsymbol{\theta}})+\boldsymbol{\tau}
$$

where $\mathbf{M}(\boldsymbol{\theta})$ is the inertia matrix, $\ddot{\boldsymbol{\theta}}$ represents the acceleration of the $i$-th link, $\mathbf{C}(\boldsymbol{\theta}, \dot{\boldsymbol{\theta}})$ are the centrifugal and Coriolis forces, $\mathbf{F}(\dot{\boldsymbol{\theta}})$ describes friction between the joint and the link and $\boldsymbol{\tau}$ is the torque effect on the rotors.

The system matrices $\mathbf{C}(\boldsymbol{\theta}, \dot{\boldsymbol{\theta}})$ and $\mathbf{F}(\dot{\boldsymbol{\theta}})$ are presented by

$$
\mathbf{C}(\boldsymbol{\theta}, \dot{\boldsymbol{\theta}})=\left[\begin{array}{ccc}
v_{11} & v_{12} & v_{13} \\
v_{21} & v_{22} & v_{23} \\
v_{31} & v_{32} & v_{33}
\end{array}\right]\left[\begin{array}{c}
\dot{\theta}_{1} \\
\dot{\theta}_{2} \\
\dot{\theta}_{M 3}
\end{array}\right] \quad \mathbf{F}(\dot{\boldsymbol{\theta}})=\left[\begin{array}{c}
k_{a} \dot{\theta}_{1} \\
k_{a} \dot{\theta}_{2} \\
b_{v} \dot{\theta}_{M 3}
\end{array}\right]
$$

where $\mathbf{v}_{3 \times 3}$ are the variables of centrifugal/coriolis forces.

The equations that define the dynamics of the DC motor, are obtained by Eq. (2.1) and Eq. (2.2), and represented by

$$
\ddot{\theta}_{M_{i}}=\frac{b_{v} \dot{\theta}_{M_{i}}-k_{t} i_{i}+\tau_{s}}{J} \quad \dot{i}_{i}=\frac{-R_{M} i_{i}-k_{b} \dot{\theta}_{M i}+V_{i}}{L_{m}}
$$

where $\ddot{\theta}_{M_{i}}$ is the acceleration and $\dot{\theta}_{M_{i}}$ the velocity of DC motor, $i_{i}$ is the current, $V_{i}$ is the voltage applied in the DC motor armature, $R_{M}, k_{b}$ and $L_{m}$ are the resistance of the armature, the electromotive force constant and the inductance of the armature of the motor, respectively.

For the coupling of the equations, we replace $\tau_{i}=\tau_{s_{i}}$ in Eqs. $(2.3),(2.4)$ and $(2.5)_{1}$, thus considering the characteristic of the proposed model flexible joints, according to (Lima et al., 2014)

$$
\boldsymbol{\tau}_{s}=\left[\begin{array}{c}
b_{s}\left(\dot{\theta}_{M_{1}}-\dot{\theta}_{1}\right)+k\left(\theta_{M 1}-\theta_{1}\right) \\
b_{s}\left(\dot{\theta}_{M_{2}}-\dot{\theta}_{2}\right)+k\left(\theta_{M_{2}}-\theta_{2}\right) \\
k i_{3}
\end{array}\right]
$$

The dynamics of the system with a flexible beam coupled to the shaft of the DC motor is given by (Janzen et al., 2014)

$$
\begin{aligned}
& \dot{i}=\frac{-R_{M} i-k_{b} \dot{\theta}+V}{L_{M}} \quad \dot{\theta}_{v}=\frac{-b_{v} \dot{\theta}+k_{t} i-C(\theta, \dot{\theta})+E I \phi_{0}^{\prime \prime} z}{J_{v}} \\
& \ddot{z}=-\mu \dot{z}-w^{2} z-\alpha_{v} \ddot{\theta}+\dot{\theta}^{2} z
\end{aligned}
$$


where $E$ is Young's modulus and $I$ is the moment of inertia of the cross section of the beam, $z$ is the displacement for the first mode of vibration of the beam and $\phi_{0}^{\prime \prime}$ is the constant of the beam vibration modes for the first mode, obtained by applying the assumed mode method (Fenili and Balthazar, 2011). Thus, the proposed system is represented by Eq. (2.3) coupled with Eq. (2.7), in the state space of the variables, represented by

$$
\begin{aligned}
\dot{x}_{1} & =x_{2} \\
\dot{x}_{2} & =p_{11}\left(-k x_{1}+k x_{3}+b_{s} x_{4}\right)+p_{12}\left(-k x_{6}+k x_{8}+b_{s} x_{9}\right)-p_{13}\left(E I \phi_{0}^{\prime \prime} x_{14}\right) \\
& +\varphi_{11} x_{2}+\varphi_{12} x_{6}+\varphi_{13} x_{11} \\
\dot{x}_{3} & =x_{4} \\
\dot{x}_{4} & =\frac{k}{J_{m}} x_{1}+\frac{b_{s}}{J_{m}} x_{2}-\frac{k}{J_{m}} x_{3}-\frac{b_{s}+b_{v}}{J_{m}} x_{4}+\frac{k_{t}}{J_{m}} x_{5} \\
\dot{x}_{5} & =-\frac{k_{b}}{L_{m}} x_{4}-\frac{R_{m}}{L_{m}} x_{5} \\
\dot{x}_{6} & =x_{7} \\
\dot{x}_{7} & =p_{21}\left(-k x_{1}+k x_{3}+b_{s} x_{4}\right)+p_{22}\left(-k x_{6}+k x_{8}+b_{s} x_{9}\right)-p_{23}\left(E I \phi_{0}^{\prime \prime} x_{14}\right) \\
& +\varphi_{21} x_{2}+\varphi_{22} x_{6}+\varphi_{23} x_{11} \\
\dot{x}_{8} & =x_{9} \\
\dot{x}_{9} & =\frac{k}{J_{m}} x_{6}+\frac{b_{s}}{J_{m}} x_{7}-\frac{k}{J_{m}} x_{8}-\frac{b_{s}+b_{v}}{J_{m}} x_{9}+\frac{k_{t}}{J_{m}} x_{10} \\
\dot{x}_{10} & =-\frac{k_{b}}{L_{m}} x_{9}-\frac{R_{m}}{L_{m}} x_{10} \\
\dot{x}_{11} & =x_{12} \\
\dot{x}_{12} & =p_{31}\left(-k x_{1}+k x_{3}+b_{s} x_{4}\right)+p_{32}\left(-k x_{6}+k x_{8}+b_{s} x_{9}\right)-p_{33}\left(E I \phi_{0}^{\prime \prime} x_{14}\right) \\
& +\varphi_{31} x_{2}+\varphi_{32} x_{6}+\varphi_{33} x_{11} \\
\dot{x}_{13} & =-\frac{k_{b}}{L_{m}} x_{14}-\frac{R_{m}}{L_{m}} x_{15} \\
\dot{x}_{14} & =x_{15} \\
\dot{x}_{15} & =-\alpha_{v} \varphi_{31} x_{12}-\alpha_{v}+\left(\omega^{2}+\alpha_{v}\left(p_{33}\left(E I \phi_{0}^{\prime \prime}+x_{12}^{2}\right)\right)\right) x_{14}-\mu x_{15}
\end{aligned}
$$

where $x_{1}=\theta_{1}, x_{2}=\dot{\theta}_{1}, x_{3}=\theta_{M_{1}}, x_{4}=\dot{\theta}_{M_{1}}, x_{5}=i_{1}, x_{6}=\theta_{2}, x_{7}=\dot{\theta}_{2}, x_{8}=\theta_{M_{2}}, x_{9}=\dot{\theta}_{M_{2}}$, $x_{10}=i_{2}, x_{11}=\theta_{M_{3}}, x_{12}=\dot{\theta}_{M_{3}}, x_{13}=i_{3}, x_{14}=z, x_{15}=\dot{z}$ and $\varphi_{n m}$ are the nonlinear elements.

The index $n$ indicates the equation of the system $\ddot{\theta}_{n}$ it belongs to, and $m$ is the corresponding coefficient of velocity $\dot{\theta}_{m}$. We can see the elements of $\varphi_{n m}$ below

$$
\begin{aligned}
& \varphi_{11}=p_{11}\left(-k_{a}-v_{11}-b_{s}\right)-p_{12} v_{21}-p_{13} v_{31} \\
& \varphi_{12}=-p_{11} v_{12}+p_{12}\left(-v_{22}-k_{a}-b_{s}\right)-p_{13} v_{32} \\
& \varphi_{13}=-p_{11} v_{13}-p_{12} v_{23}+p_{13}\left(-v_{33}-b_{v}\right) \\
& \varphi_{21}=p_{21}\left(-k_{a}-v_{11}-b_{s}\right)-p_{22} v_{21}-p_{23} v_{31} \\
& \varphi_{22}=-p_{21} v_{12}+p_{12}\left(-v_{22}-k_{a}-b_{s}\right)-p_{23} v_{32} \\
& \varphi_{23}=-p_{21} v_{13}-p_{12} v_{23}+p_{23}\left(-v_{33}-b_{v}\right) \\
& \varphi_{31}=p_{31}\left(-k_{a}-v_{11}-b_{s}\right)-p_{32} v_{21}-p_{33} v_{31} \\
& \varphi_{32}=-p_{31} v_{12}+p_{32}\left(-v_{22}-k_{a}-b_{s}\right)-p_{33} v_{32} \\
& \varphi_{33}=-p_{31} v_{13}-p_{32} v_{23}+p_{33}\left(-v_{33}-b_{v}\right)
\end{aligned}
$$

and

$$
\mathbf{p}_{3 \times 3}=\mathbf{M}(\boldsymbol{\theta})^{-1}
$$




\section{Proposed control}

Consider system (2.8) in the form

$$
\dot{\mathbf{x}}=\mathbf{A}(\mathbf{x}) \mathbf{x}+\mathbf{B u}
$$

where $\mathbf{x} \in \Re^{n}$ is the state vector, $\mathbf{A}(\mathbf{x}) \in \Re^{n \times n}$ is the state-dependent matrix, $\mathbf{B} \in \Re^{n \times m}$ is the control matrix and $\mathbf{u}$ is a feedback control vector.

The proposed control $\mathbf{u}$ can be determined using SDRE control (Balthazar et al., 2014). The methodology used in SDRE control makes the synthesis of LQR control gains varying in time (Mracek and Cloutier, 1998). The quadratic cost function for the regulator problem is given by

$$
J=\frac{1}{2} \int_{t_{0}}^{\infty}\left[\mathbf{e}^{\mathrm{T}} \mathbf{Q e}+\mathbf{u}^{\mathrm{T}} \mathbf{R u}\right] d t
$$

where $\mathbf{Q} \in \Re^{n \times n}$ and $\mathbf{R} \in \Re^{m \times m}$ are positive definite matrices, and

$$
\mathbf{e}=\left[\begin{array}{lllllllllllllll}
x_{1}-x_{1}^{*} & 0 & 0 & 0 & 0 & x_{2}-x_{2}^{*} & 0 & 0 & 0 & 0 & x_{3}-x_{3}^{*} & 0 & 0 & 0 & 0
\end{array}\right]^{\mathbf{T}}
$$

where $\mathbf{x}$ are the system state variables, and $x^{*}$ is the desired state.

Assuming full state feedback, the control law is given by

$$
\mathbf{u}=-\mathbf{R}^{-1} \mathbf{B}^{\mathrm{T}} \mathbf{P e}
$$

The State-Dependent-Riccati equation to obtain $\mathbf{P}$ is given by

$$
\mathbf{P}(\mathbf{x}) \mathbf{A}(\mathbf{x})+\mathbf{A}(\mathbf{x})^{\mathrm{T}} \mathbf{P}(\mathbf{x})-\mathbf{P}(\mathbf{x}) \mathbf{B} \mathbf{R}^{-1} \mathbf{B}^{\mathrm{T}} \mathbf{P}(\mathbf{x})+\mathbf{Q}=\mathbf{0}
$$

In numerical simulations, we consider two situations. First, we consider control of the handler via DC motors at the joints without control of vibrations of the flexible beam. Next, we consider control of the vibration of the flexible beam. It is assumed throughout this paper that the parameters considered are presented in Table 1.

Table 1. Parameters for simulation

\begin{tabular}{|l|l|l|}
\hline$L_{1}=0.5 \mathrm{~m}$ & $L_{2}=0.5 \mathrm{~m}$ & $L_{3}=1.2 \mathrm{~m}$ \\
$M_{1}=1 \mathrm{~kg}$ & $M_{2}=1 \mathrm{~kg}$ & $M_{3}=1.318 \cdot 10^{-4} \mathrm{~kg}$ \\
$k_{t 1}=0.08 \mathrm{Nm} / \mathrm{A}$ & $k_{t 2}=0.08 \mathrm{Nm} / \mathrm{A}$ & $k_{t 3}=0.058 \mathrm{Nm} / \mathrm{A}$ \\
$k_{b 1}=0.008 \mathrm{~V} / \mathrm{rpm}$ & $k_{b 2}=0.008 \mathrm{~V} / \mathrm{rpm}$ & $k_{b 3}=0.008 \mathrm{~V} / \mathrm{rpm}$ \\
$b_{v 1}=0.2 \mathrm{Nms} / \mathrm{rad}$ & $b_{v 2}=0.2 \mathrm{Nms} / \mathrm{rad}$ & $b_{v 3}=4.62 \mathrm{Nms} / \mathrm{rad}$ \\
$R_{m 1}=1.7 \Omega$ & $R_{m 2}=1.7 \Omega$ & $R_{m 3}=1.914 \Omega$ \\
$L_{m 1}=0.003 \mathrm{H}$ & $L_{m 2}=0.003 \mathrm{H}$ & $L_{m 3}=3.4 \cdot 10^{-3} \mathrm{H}$ \\
$E=0.7 \cdot 10^{11} \mathrm{~N} / \mathrm{m}^{2}$ & $I_{m}=6.54 \cdot 10^{-5} \mathrm{~kg} / \mathrm{m}^{2}$ & $I_{e}=6.54 \cdot 10^{-7} \mathrm{~kg} / \mathrm{m}^{2}$ \\
$I=1.56 \cdot 10^{-13} \mathrm{~m}^{4}$ & $\mu=0.1$ & $\phi_{0}^{\prime \prime}=4.898 \mathrm{~m}$ \\
$\alpha_{v}=0.821$ & $w=11.3097 \mathrm{rad} / \mathrm{s}$ & \\
\hline
\end{tabular}

The initial values for $\mathbf{x}$ are given by: $x_{1}(0)=\pi, x_{2}(0)=0, x_{3}(0)=\pi, x_{4}(0)=0, x_{5}(0)=0$, $x_{6}(0)=0.6981, x_{7}(0)=0, x_{8}(0)=0.6981, x_{9}(0)=0, x_{10}(0)=0, x_{11}(0)=0.5236, x_{12}(0)=0$, $x_{13}(0)=0, x_{14}(0)=0$ and $x_{15}(0)=0$.

The desired state is: $x_{1}^{*}=2.0944, x_{2}^{*}=0, x_{3}^{*}=x_{3}, x_{4}^{*}=x_{4}, x_{5}^{*}=x_{5}, x_{6}^{*}=-0.6981, x_{7}^{*}=0$, $x_{8}^{*}=x_{8}, x_{9}^{*}=x_{9}, x_{10}^{*}=x_{10}, x_{11}^{*}=-0.5236, x_{12}^{*}=0, x_{13}^{*}=x_{13}, x_{13}(0)=0, x_{14}^{*}=0$ and $x_{15}^{*}=0$. 


\subsection{Position control for DC motor (control I)}

Consider the case in which the control $\mathbf{u}$ is applied only to position control of $\theta_{1}, \theta_{2}$ and $\theta_{M 3}$, and flexible link vibrations are not controlled directly. Thus, we define $\mathbf{Q}, \mathbf{R}=\mathbf{R}_{1}$ and consider $\mathbf{u}=\left[\begin{array}{lll}u_{1} & u_{2} & u_{3}\end{array}\right]^{\mathrm{T}}, \mathbf{A}=\left[\begin{array}{ll}\mathbf{A}_{1} & \mathbf{A}_{2}\end{array}\right], \mathbf{B}=\mathbf{B}_{1}$, where the control signal $\left(u_{1}, u_{2}\right.$ and $\left.u_{3}\right)$ represents the voltage applied to the motor terminals (matrices $\mathbf{Q}, \mathbf{R}_{1}, \mathbf{A}_{1}, \mathbf{A}_{2}$ and $\mathbf{B}_{1}$ in Appendix). In Figs. 2a,b,c, we can observe the position error of the manipulator $\left(\theta_{1}, \theta_{2}\right.$ and $\left.\theta_{M 3}\right)$, considering only position control of the link by the motor current. In Fig. 2d, we can observe vibrations at the tip of the flexible link $(z)$.

(a)

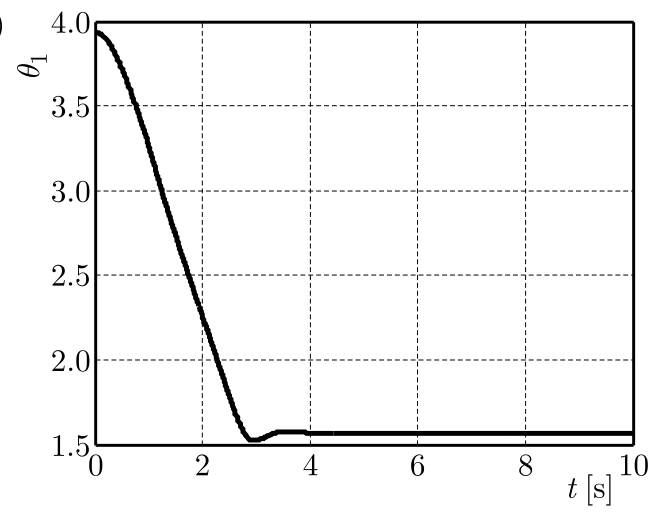

(c)

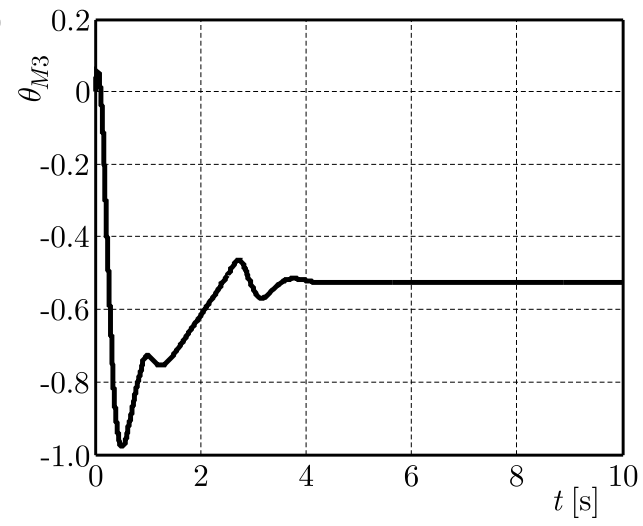

(b)

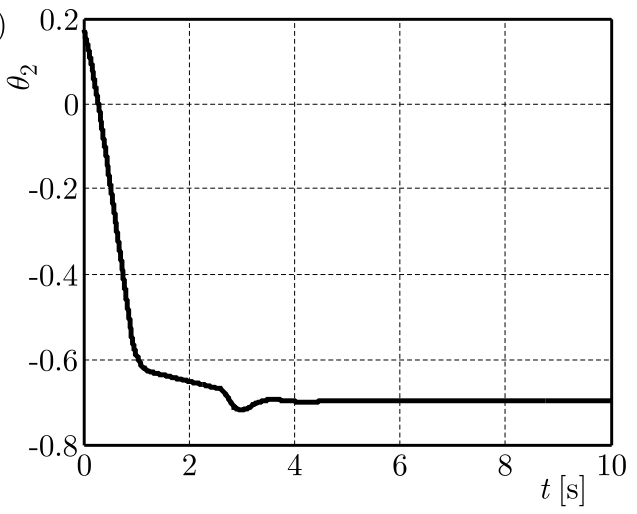

(d)

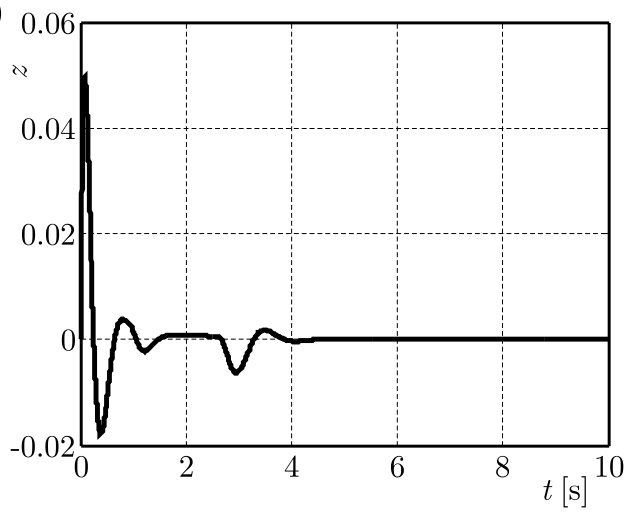

Fig. 2. (a) Positioning $\theta_{1}$, (b) positioning $\theta_{2}$, (c) positioning motor $\theta_{M 3}$, (d) displacement of the beam $z$

Although the system controlled by the motors can position the links at the desired points, we observe oscillations in the positioning (Fig. 2a,b,c) caused by vibrations of the flexible link (Fig. 2d).

\subsection{Position control for DC motor and beam deflection control (control II)}

In the adopted second strategy we control $\theta_{1}, \theta_{2}$ and $\theta_{M 3}$ and control the flexible link vibrations using SMAs fixed to the link. SMAs capacity to change their physical properties with temperature variation allows for their use as means to vibration suppression of the flexible link.

Falk (1980, 1993) proposed the SMA mathematical model used in this paper. It is based on the Devonshire theory that considers free energy in a polynomial form. This model does not consider internal variables and potential dissipation to describe the pseudo-elasticity and the shape memory effect. The Falk model considers only the temperature $T$ and the deformation $(\varepsilon)$ (Janzen et al., 2014).

The SMA polynomial model was chosen due to its capacity to represent qualitatively the pseudo-elasticity and shape memory effects of the SMA structure. The Helmholtz free energy $\psi$ 
was chosen so there are minimum and maximum points that represent the stability and instability of the SMA phases. The potential of free energy can be described by

$$
\rho \psi(\varepsilon, T)=\frac{1}{2} q\left(T-T_{M}\right) \varepsilon^{2}-\frac{1}{4} b \varepsilon^{4}+\frac{b^{2} \varepsilon^{6}}{24 q\left(T_{A}-T_{M}\right)}
$$

The variables $T_{A}$ and $T_{M}$ are temperatures in which the austenitic and martensitic phases are stable, $q$ and $b$ are positive constants. A constitutive equation can be written as (Savi et al., 2002)

$$
\sigma=q\left(T-T_{M}\right) \varepsilon-\frac{1}{4} b \varepsilon^{3}+\frac{b^{2} \varepsilon^{5}}{4 q\left(T_{A}-T_{M}\right)}
$$

where $b=1.868 \cdot 10^{7} \mathrm{MPa}, q=523.29 \mathrm{MPa} / \mathrm{K}, T_{M}=364.3 \mathrm{~K}$ and $T_{M}=288 \mathrm{~K}$ for $(\mathrm{Cu}-\mathrm{Zn}-\mathrm{Al}-\mathrm{Ni})$.

The SMA element can be coupled to the beam-like structure as a stiffness component which can be described by

$$
k(x, T)=\bar{q}\left(T-T_{M}\right) x-\bar{b} x^{3}+\bar{e} x^{5}
$$

where

$$
\bar{q}=\frac{q A_{r}}{L} \quad \bar{b}=\frac{b A_{r}}{L^{3}} \quad \bar{e}=\frac{e A_{r}}{L^{5}}
$$

Considering the case in which the objective is to position the beam-like link and reducing its oscillations, we define $\mathbf{Q}, \mathbf{R}=\mathbf{R}_{2}$ and consider $\mathbf{u}_{f}=\left[\begin{array}{llll}u_{1} & u_{2} & u_{3} & u_{4}\end{array}\right]^{\mathrm{T}}, \mathbf{A}=\left[\begin{array}{ll}\mathbf{A}_{1} & \mathbf{A}_{3}\end{array}\right]$ and $\mathbf{B}=\mathbf{B}_{2}$ (matrices $\mathbf{Q}, \mathbf{R}_{2}, \mathbf{A}_{1}, \mathbf{A}_{3}$ and $\mathbf{B}_{2}$ in Appendix). And considering: $\bar{q}=1.56987, \bar{b}=114367.348$ and $\bar{e}=7232491.36$ (Janzen et al., 2014).

The control signal $\left(u_{1}, u_{2}\right.$ and $\left.u_{3}\right)$ represents the voltage applied to the motor terminals, and $\left(u_{4}\right)$ represents the temperature variation of the SMA given by

$$
u_{4}=\Delta T=T-T_{M}
$$

In Figs. 3a,b and 3c, we can observe the manipulator position error $\left(\theta_{1}, \theta_{2}\right.$ and $\left.\theta_{M 3}\right)$ considering the positioning control of links through the motors, and the vibration control of flexible link through the use of SMA in Fig. 3d $(z)$.

As we can see in Fig. 3, the addition of SMA reduces vibrations of the flexible link, therefore reducing oscillations $\left(\theta_{M 3}\right)$. Thus, we avoid inversion of the torques of the motors, which caused the oscillations seen in Fig. 2.

In Fig 4a, we can observe the temperature variation of the SMA to control the vibration of the flexible link $(\Delta T)$, and in Fig. 4b we present the comparative results between the two control strategies proposed.

First, the structure is fully austenite, and it can be seen from Fig. 4 that no phase transformation occurs in the alloy, namely, the SMA remains in the austenite phase $\left(T_{A} \geqslant 364.3 \mathrm{~K}\right)$. This variation of the parameter $\Delta T$ acts on the beam rigidity (Eq. (3.8)) and reduces the amplitude of displacement of the flexible link as can be observed in Fig. 4b.

It is also possible to observe that $\Delta T$ remained at a constant value of $\Delta T=77 \mathrm{~K}$ for $t>0.029 \mathrm{~s}$, due to the need of keeping the SMA in the austenite phase, enables control of the stiffness according to equation (3.8).

By applying DC control motors and using flexible SMA one observes a smoother response for all angles $\theta_{1}, \theta_{2}$ and $\theta_{M 3}$ and a smaller amplitude of oscillation $z$. 
(a)

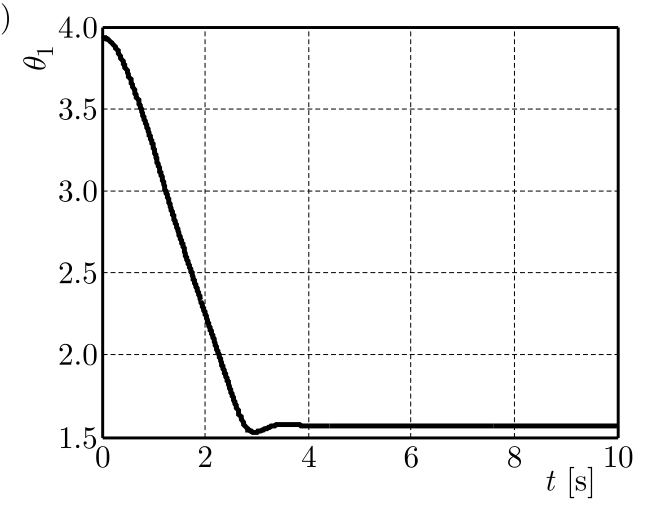

(c)

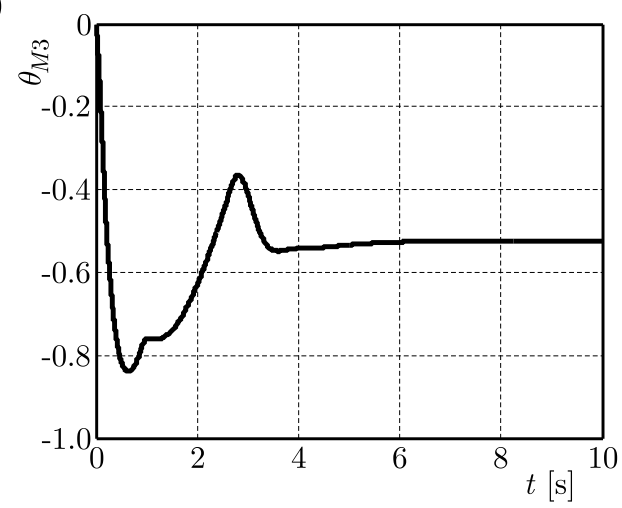

(b)

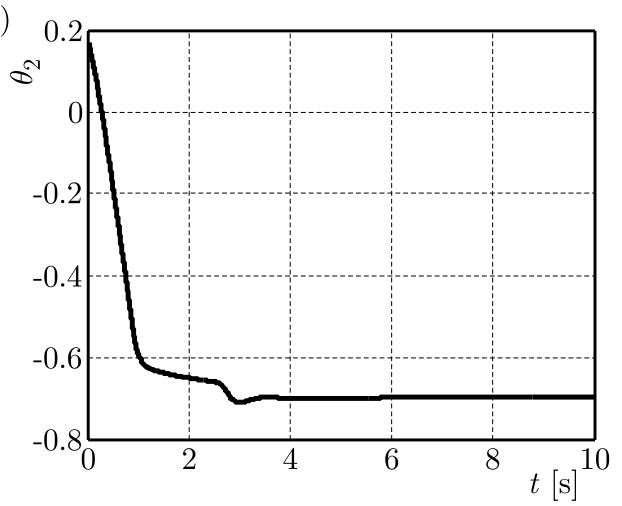

(d)

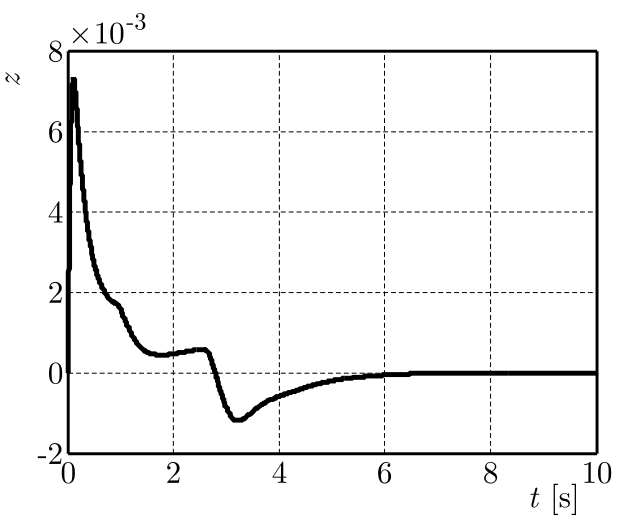

Fig. 3. (a) Positioning $\theta_{1}$, (b) positioning $\theta_{2}$, (c) positioning motor $\theta_{M 3}$, (d) displacement of the beam $z$

(a)

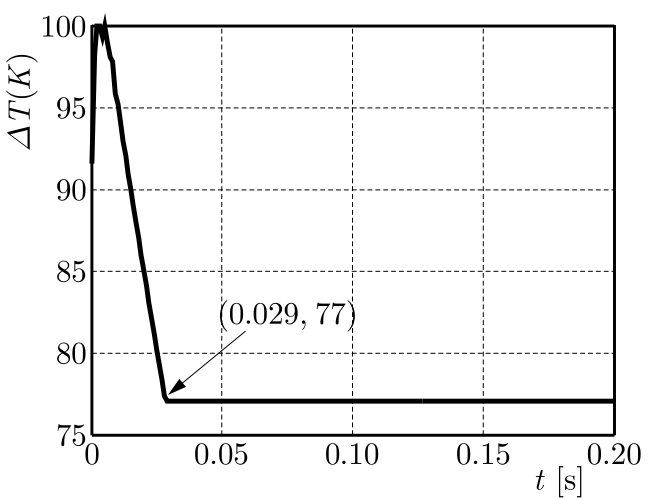

(b)

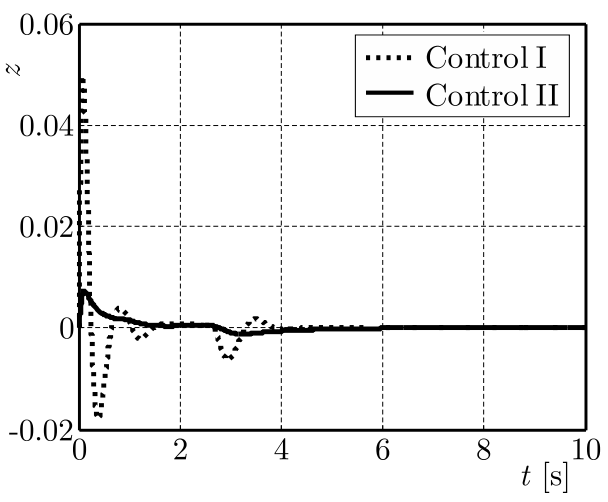

Fig. 4. (a) Temperature variation in SMA $\left(u_{4}=T-288=\Delta T\right)$, (b) displacement of the beam $z$

\section{Conclusion}

We can observe in the simulations results that vibration control of the flexible link allows for a more efficient control than the control provided by the motors only. This demonstrates the importance of controlling vibrations of the flexible link. By using the polynomial model of SMA it is possible to estimate the temperature variation in the SMA to attenuate vibrations of the flexible link. The temperature variation of the SMA causes changes in their physical characteristics, allowing for its use as a way to reduce vibrations of the flexible beam. The numerical results show that the proposed control strategy using SMA in vibration control of the flexible links is a good alternative to constructing lighter handlers with longer links, such as those applied in aerospace activities. 


\section{Acknowledgements}

The authors would like to acknowledge Conselho Nacional de Desenvolvimento Cientfico e Tecnológico - CNPQ grant: 484729/2013-6 and grant: 447539/2014-0, for the financial support.

\section{Appendix}

$$
\begin{aligned}
& \mathbf{A}_{1}=\left[\begin{array}{cccccccccc}
0 & 1 & 0 & 0 & 0 & 0 & 0 & 0 & 0 & 0 \\
-p_{11} k & \varphi_{11} & p_{11} k & p_{11} b_{s} & 0 & -p_{12} k & \varphi_{12} & p_{12} k & p_{12} b_{s} & 0 \\
0 & 0 & 0 & 1 & 0 & 0 & 0 & 0 & 0 & 0 \\
\frac{k}{J_{m}} & \frac{b_{s}}{J_{m}} & -\frac{k}{J_{m}} & -\frac{b_{s}+b_{v}}{J_{m}} & \frac{k_{t}}{J_{m}} & 0 & 0 & 0 & 0 & 0 \\
0 & 0 & 0 & 0 & 0 & 0 & 1 & 0 & 0 & 0 \\
0 & 0 & 0 & -\frac{k_{b}}{L_{m}} & -\frac{R_{m}}{L_{m}} & 0 & 0 & 0 & 0 & 0 \\
-p_{21} k & \varphi_{21} & p_{21} k & p_{21} b_{s} & 0 & -p_{22} k & \varphi_{22} & p_{22} k & p_{22} b_{s} & 0 \\
0 & 0 & 0 & 0 & 0 & 0 & 0 & 0 & 1 & 0 \\
0 & 0 & 0 & 0 & 0 & \frac{k}{J_{m}} & \frac{b_{s}}{J_{m}} & -\frac{k}{J_{m}} & -\frac{b_{s}+b_{v}}{J_{m}} & \frac{k_{t}}{J_{m}} \\
0 & 0 & 0 & 0 & 0 & 0 & 0 & 0 & -\frac{k_{b}}{L_{m}} & -\frac{R_{m}}{L_{m}} \\
0 & 0 & 0 & 0 & 0 & 0 & 0 & 0 & 0 & 0 \\
-p_{31} k & \varphi_{31} & p_{31} k & p_{31} b_{s} & 0 & -p_{32} k & \varphi_{32} & p_{32} k & p_{32} b_{s} & 0 \\
0 & 0 & 0 & 0 & 0 & 0 & 0 & 0 & 0 & 0 \\
0 & 0 & 0 & 0 & 0 & 0 & 0 & 0 & 0 & 0 \\
0 & 0 & 0 & 0 & 0 & 0 & 0 & 0 & 0 & 0
\end{array}\right] \\
& \mathbf{B}_{1}^{\mathrm{T}}=\left[\begin{array}{ccccccccccccccc}
0 & 0 & 0 & \frac{1}{L_{M}} & 0 & 0 & 0 & 0 & 0 & 0 & 0 & 0 & 0 & 0 & 0 \\
0 & 0 & 0 & 0 & 0 & 0 & 0 & 0 & 0 & \frac{1}{L_{M}} & 0 & 0 & 0 & 0 & 0 \\
0 & 0 & 0 & 0 & 0 & 0 & 0 & 0 & 0 & 0 & 0 & 0 & \frac{1}{L_{M}} & 0 & 0
\end{array}\right]
\end{aligned}
$$$$
\mathbf{Q}_{15 \times 15}=\left\{q_{i j}\right\} \quad q_{i j}= \begin{cases}0 & \text { if } \quad i \neq j \\ 1 & \text { if } i=2,3,4,5,7,8,9,10,12,13,15 \\ 100 & \text { if } i=1,6,11,14\end{cases}
$$$$
\mathbf{R}_{1}=\left[\begin{array}{ccc}
0.1 & 0 & 0 \\
0 & 0.1 & 0 \\
0 & 0 & 0.1
\end{array}\right] \quad \mathbf{R}_{2}=\left[\begin{array}{cccc}
0.1 & 0 & 0 & 0 \\
0 & 0.1 & 0 & 0 \\
0 & 0 & 0.1 & 0 \\
0 & 0 & 0 & 0.1
\end{array}\right]
$$

$$
\mathbf{A}_{2}=\left[\begin{array}{ccccc}
0 & 0 & 0 & 0 & 0 \\
0 & \varphi_{13} & p_{13} k_{t} & -p_{13} E I \phi_{0}^{\prime \prime} & 0 \\
0 & 0 & 0 & 0 & 0 \\
0 & 0 & 0 & 0 & 0 \\
0 & 0 & 0 & 0 & 0 \\
0 & 0 & 0 & 0 & 0 \\
0 & \varphi_{23} & p_{23} k_{t} & -p_{23} E I \phi_{0}^{\prime \prime} & 0 \\
0 & 0 & 0 & 0 & 0 \\
0 & 0 & 0 & 0 & 0 \\
0 & 0 & 0 & 0 & 0 \\
0 & 0 & 0 & 0 & 0 \\
0 & \varphi_{33} & p_{33} k_{t} & -p_{33} E I \phi_{0}^{\prime \prime} & 0 \\
0 & -\frac{k_{b}}{L_{M}} & -\frac{R_{M}}{L_{M}} & 0 & 0 \\
0 & 0 & 0 & 0 & 1 \\
0 & -\alpha_{v} \varphi_{33} & \alpha_{v} p_{33} k_{t} & w^{2}+\alpha_{v} p_{33} E I \phi_{0}^{\prime \prime}+x_{12}^{2} & \mu
\end{array}\right]
$$




$$
\begin{aligned}
& \mathbf{B}_{2}=\left[\begin{array}{cccc}
0 & 0 & 0 & 0 \\
0 & 0 & 0 & 0 \\
0 & 0 & 0 & 0 \\
\frac{1}{L_{M}} & 0 & 0 & 0 \\
0 & 0 & 0 & 0 \\
0 & 0 & 0 & 0 \\
0 & 0 & 0 & 0 \\
0 & 0 & 0 & 0 \\
0 & 0 & 0 & 0 \\
0 & \frac{1}{L_{M}} & 0 & 0 \\
0 & 0 & 0 & 0 \\
0 & 0 & 0 & 0 \\
0 & 0 & \frac{1}{L_{M}} & 0 \\
0 & 0 & 0 & 0 \\
0 & 0 & 0 & -\bar{q} x_{14}
\end{array}\right] \\
& \mathbf{A}_{3}=\left[\begin{array}{ccccc}
0 & 0 & 0 & 0 & 0 \\
0 & \varphi_{13} & p_{13} k_{t} & -p_{13} E I \phi_{0}^{\prime \prime} & 0 \\
0 & 0 & 0 & 0 & 0 \\
0 & 0 & 0 & 0 & 0 \\
0 & 0 & 0 & 0 & 0 \\
0 & 0 & 0 & 0 & 0 \\
0 & \varphi_{23} & p_{23} k_{t} & -p_{23} E I \phi_{0}^{\prime \prime} & 0 \\
0 & 0 & 0 & 0 & 0 \\
0 & 0 & 0 & 0 & 0 \\
0 & 0 & 0 & 0 & 0 \\
0 & 0 & 0 & -p_{33} E I \phi_{0}^{\prime \prime} & 0 \\
0 & \varphi_{33} & p_{33} k_{t} & 0 & 0 \\
0 & -\frac{k_{b}}{L_{M}} & -\frac{R_{M}}{L_{M}} & 0 & 0 \\
0 & 0 & 0 & & 1 \\
0 & -\alpha_{v} \varphi_{33} & \alpha_{v} p_{33} k_{t} & w^{2}+\alpha_{v} p_{33} E I \phi_{0}^{\prime \prime}+x_{12}^{2}-x_{14}^{2}\left(\bar{b}-\bar{e} x_{14}^{2}\right) & \mu
\end{array}\right]
\end{aligned}
$$

\section{References}

1. Balthazar J.M., Bassinello D.G., Tusset A.M., Bueno A.M., Pontes B.R., 2014, Nonlinear control in an electromechanical transducer with chaotic behavior, Meccanica, 49, 8, 1859-1867, doi: 10.1007/s11012-014-9910-4

2. Banks H.T., Smith R.C., Wang Y., 1996, Smart Material Structures: Modeling, Estimation and Control, John Wiley \& Sons, Paris

3. Bottega V., Pergher R., Fonseca J.S.O., 2009, Simultaneous control and piezoelectric insert optimization for manipulators with flexible link, Journal of the Brazilian Society of Mechanical Sciences and Engineering, 31, 2, 105-116, doi: 10.1590/S1678-58782009000200003

4. FAlK F., 1980, Model free energy, mechanics, and thermodynamics of shape memory alloys, Acta Metallurgica, 28, 12, 1773-1780, doi: 10.1016/0001-6160(80)90030-9

5. FALK F., 1983, One dimensional model of shape memory alloys, Archives of Mechanics, 35, 1, 63-84.

6. Fenili A., Balthazar J.M., 2011, The rigid-flexible nonlinear robotic manipulator: modeling and control, Communications in Nonlinear Science and Numerical Simulation, 16, 5, 2332-2341, doi: 10.1016/j.cnsns.2010.04.057 
7. Gabbert U., Schulz I., 1996, Optimal placement of piezoelectric actuators in vibration control of adaptative structures, Proceedings of ASME International Mechanical Engineering Congress and Exposition, 271-277

8. Ge S.S., Tee K.P., VAhHi I.E., TAY F.E.H., 2006, Tracking and vibration control of flexible robots using shape memory alloys, IEEE/ASME Transactions on Mechatronics, 11, 690-698, doi: 10.1109/TMECH.2006.886242

9. Grandinetti F.J., Soares A.M.S., Lamas W.Q., Goes L.C.S., 2012, Notes on vibration control of a micro/macromanipulator mounted on a flexible structure, Proceedings of the Institution of Mechanical Engineers, Part K: Journal of Multi-body Dynamics March, 226, 1, 72-82, doi: $10.1177 / 1464419311424090$

10. Halim D., Luo X., Trivailo P.M., 2014, Decentralized vibration control of a multi-link-flexible robotic manipulator using smart piezoelectric transducers, Acta Astronautica, 104, 186-196, doi: 10.1016/j.actaastro.2014.07.016

11. Heidari H.R., Korayem M.H., Haghpanahi M., Batlle V.F., 2013, Optimal trajectory planning for flexible link manipulators with large deflection using a new displacements approach, Journal of Intelligent and Robotic Systems, 72, 3/4, 287-300, doi: 10.1007/s10846-012-9807-1

12. Innocenti M., Baralli F., Salotti F., Caiti A., 2000, Manipulator path control using SDRE, American Control Conference, 5, 3348-3352, doi: 10.1109/ACC.2000.879186

13. Janzen F.C., Tusset A.M., Piccirillio V., Balthazar J.M., Silveira M., Pontes Junior B.R., 2014, Control of slewing motions of flexible structures using shape memory alloy actuators, Proceedings of ASME International Mechanical Engineering Congress and Exposition, 1-7

14. JNIFEnE A., 2007, Active vibration control of flexible structures using delayed position feedback, Systems and Control Letters, 56, 3, 215-222, doi: 10.1016/j.sysconle.2006.10.005

15. Kalyoncu M., 2008, Mathematical modelling and dynamic response of a multistraight-line path tracing flexible robot manipulator with rotating-prismatic joint, Applied Mathematical Modelling, 32, 1087-1098, doi: 10.1016/j.apm.2012.10.042

16. Korayem M.H., Irani M., Nekoo S.R., 2010, Analysis of manipulators using SDRE: a closed loop nonlinear optimal control approach, Scientia Iranica, Transaction B: Mechanical Engineering, 17, 6, 456-467

17. Korayem M., Irani M., Nekoo S.R., 2011, Load maximization of flexible joint mechanical manipulator using nonlinear optimal controller, Acta Astronautica, 69, 7, 458-469, doi: 10.1016/j.actaastro.2011.05.023

18. Korayem M.H., Nekoo S.R., 2014, Nonlinear optimal control via finite time horizon statedependent Riccati equation, International Conference on Robotics and Mechatronics, 878-883, doi: 10.1109/ICRoM.2014.6991015

19. Korayem M.H., Nekoo S.R., 2015a, State-dependent differential Riccati equation to track control of time-varying systems with state and control nonlinearities, ISA Transactions, 57, 117-135, doi: $10.1016 /$ j.isatra.2015.02.008

20. Korayem M.H., Nekoo S.R., 2015b, Finite-time state-dependent Riccati equation for timevarying nonaffine systems: rigid and flexible joint manipulator control, ISA Transactions, 54, 125144, doi:10.1016/j.isatra.2014.06.006

21. Lima J.J., Tusset A.M., Janzen F.C., Piccirillo V., Nascimento C.B., Balthazar J.M., BrasiL M.R.L.F., 2014, Nonlinear state estimation and control applied to a manipulator robotic including drive motor, Mathematics in Engineering, Science and Aerospace: the Transdisciplinary International Journal, 5, 413-425

22. Mracek P.C., Cloutier J.R., 1998, Control designs for the nonlinear benchmark problem via the state-dependent Riccati equation method, International Journal of Robust and Nonlinear Control, 8, 401-433 
23. Molter A., Silveira O.A.A., Fonseca J.S.O., Bottega V., 2010, Simultaneous piezoelectric actuator and sensor placement optimization and control design of manipulators with flexible links using SDRE method, Mathematical Problems in Engineering, 1-23, doi: 10.1155/2010/362437

24. NEKOo S.R., 2013, Nonlinear closed loop optimal control: a modified state-dependent Riccati equation, ISA Transactions, 52, 2, 285-290, doi:10.1016/j.isatra.2012.10.005

25. Pereira E., Trapero J.R., Diaz I.M., Feliu V., 2012, Adaptive input shaping for single-link flexible manipulators using an algebraic identification, Control Engineering Practice, 20, 138-147, doi: $10.1016 /$ j.conengprac.2011.10.004

26. Piccirillo V., Balthazar J.M., Pontes Jr. B.R., Felix J.L.P., 2008, On a nonlinear and chaotic non-ideal vibrating system with Shape Memory Alloy (SMA), Journal of Theoretical and Applied Mechanics (Poland), 46, 597-620

27. Piccirillo V., Balthazar J.M., Pontes Jr. B.R., Felix J.L.P., 2009, Chaos control of a nonlinear oscillator with shape memory alloy using an optimal linear control. Part II: Non-ideal energy source, Nonlinear Dynamics, 56, 3, 243-253, doi: 10.1007/s11071-008-9396-5

28. Savi M.A., Pacheco P.M.C.L., Braga A.M.B., 2002, Chaos in a shape memory two-bars truss, International Journal of Non-Linear Mechanics, 37, 1387-1395

29. Sawada H., Ui K., Mori M., Yamamoto H., Hayashi R., Matunaga S., Ohkami Y., 2004, Micro-gravity experiment of a space robotic arm using parabolic flight, Advanced Robotics, 18, 3, 247-267, doi: 10.1163/156855304322972431

30. Shawky A., Zydek D., Elhalwagy Y.Z., Ordys A., 2013, Modeling and nonlinear control of a flexible-link manipulator, Applied Mathematical Modelling, 37, 9591-9602, doi: 10.1016/j.apm.2013.05.003

31. Xin M., Balakrishnan S.N., Huang Z., 2001, Robust state dependent Riccati equation based robot manipulator control, International Conference on Control Applications, 3, 369-374, doi: 10.1109/CCA.2001.973893 